\title{
REMARKS ABOUT A CLOSURE ALGEBRA IN WHICH CLOSED ELEMENTS ARE OPEN
}

\author{
JEAN E. RUBIN
}

The closure algebra ${ }^{1}$ which is considered here is one in which the closure operation $C$ satisfies the condition:

$$
-C-C x=C x \text { for all } x \text { in the domain of } C .
$$

A closure algebra which satisfies (1) will be called a C-5 algebra. ${ }^{2}$ In $\$ 1$, it is shown that condition (1) may be expressed in many diverse forms. For example, in a closure algebra, (1) is equivalent to:

$$
C(x \cdot C y)=C x \cdot C y \text { for all } x \text { and } y \text { in the domain of } C .
$$

It is also shown that the closure operator in a C-5 algebra is completely distributive over addition. In $\$ 2$, MacNeille's extension ${ }^{3}$ of a C-5 algebra is defined and it is shown that the extension is a C-5 algebra.

1. C-5 algebras. A C-5 algebra is defined as follows:

Definition 1.1. An ordered quadruple $A=\langle A, \cdot,-, C\rangle$ is said to be a $\mathrm{C}-5$ algebra if the following conditions are satisfied:

(i) $\langle A, \cdot,-\rangle$ is a Boolean algebra. (We shall use + as the Boolean operation of addition; $\leqq$ as the Boolean relation of less than or equal to; 0 and 1 as the zero and unit elements of the Boolean algebra.)

(ii) $C$ is a unary function from $A$ to $A$.

(iii) $x \leqq C x$ for all $x$ in $A$.

(iv) $C C x=C x$ for all $x$ in $A$.

(v) $C(x+y)=C x+C y$ for all $x$ and $y$ in $A$.

(vi) $C 0=0$.

(vii) $-C-C x=C x$ for all $x$ in $A$.

Remark. The algebra $\mathcal{A}$ of 1.1 is said to be a closure algebra if it satisfies (i)-(vi).

Received by the editors January 18, 1955.

${ }^{1}$ For a definition of a closure algebra and a discussion of its properties see McKinsey and Tarski [3].

2 The name "C-5 algebra" is used because the algebra is an algebraic model (characteristic matrix) for Lewis' system of modal logic S5. A closure algebra is an algebraic model for Lewis' system S4. For a definition of S4 and S5 see Lewis and Langford [2], Appendix II.

${ }^{3}$ For a definition of MacNeille's extension of a partially ordered set see MacNeille $[4$, p. $443 \mathrm{ff}$.$] .$ 
The interior of an element $x, I x$, is defined to be the complement of the closure of the complement of $x,-C-x$. Therefore, condition (vii) of 1.1 means that the interior of the closure of $x$ is the closure of $x$. Furthermore, taking the complement of both sides of equation (vii) and substituting $-x$ for $x$, we obtain:

$$
C-C-x=-C-x \text { for all } x \text { in } A \text {. }
$$

That is, the closure of the interior of $x$ is the interior of $x$. It is clear that (2) is equivalent to (vii). The following theorem gives further conditions which are equivalent to (vii).

Theorem 1.2. If $A=\langle A, \cdot,-, C\rangle$ is a closure algebra then the following conditions are equivalent:

(1) $-C-C x=C x$ for all $x$ in $A$.

(2) $C-C-x=-C-x$ for all $x$ in $A$.

(3) $C(x \cdot C y)=C x \cdot C y$ for all $x$ and $y$ in $A$.

(4) If $C x=x$ then $C-x=-x$ for all $x$ in $A$.

(5) If $x \cdot C y=0$ then $C x \cdot C y=0$ for all $x$ and $y$ in $A$.

(6) If $x \cdot C y=0$ then $C x \cdot y=0$ for all $x$ and $y$ in $A$.

REMARKs. An element $x$ is said to be closed if $C x=x$ and open if $I x=x$. An element is closed if and only if its complement is open. Hence it follows from (4) that in a C-5 algebra, an element is closed if and only if it is open and also that the set of closed elements (which is the same as the set of open elements) forms a Boolean algebra under the operations of $\cdot$ and - . Condition (6) is the condition that $C$ is a self-conjugate function. ${ }^{4}$ Therefore, the operator $C$ in a C-5 algebra satisfies the same properties as each of the cylindrification operators in a cylindric algebra. ${ }^{5}$

Proof of Theorem 1.2. We have previously shown that (1) is equivalent to (2). To carry out the remaining part of the proof we shall show (a) (1) implies (3), (b) (3) implies (5), (c) (5) implies (6), (d) (6) implies (4), and finally (e) (4) implies (1).

(a) Using Boolean algebraic properties, it follows that:

$$
x+(-C y)=x \cdot C y+(-C y) ;
$$

then, taking the closure of both sides of this equation and utilizing $1.1(\mathrm{v})$ :

$$
C x+C-C y=C(x \cdot C y)+C-C y .
$$

Now, from (1) it follows that $C-C y=-C y$, hence:

'Jonsson and Tarski [1, p. 903].

s Ibid. p. 936. 


$$
C x+(-C y)=C(x \cdot C y)+(-C y) .
$$

Multiplying both sides of the preceding equation by $C y$ and simplifying, we obtain:

$$
C x \cdot C y=C(x \cdot C y) \cdot C y .
$$

But from 1.1 (iii), (iv), and (v) it follows that $C(x \cdot C y) \leqq C y$, so that $C(x \cdot C y) \cdot C y=C(x \cdot C y)$. Therefore, (1) implies (3).

(b) Assume $x \cdot C y=0$, then it follows from (3) that $C x \cdot C y=C 0$. But, by 1.1 (vi), $C 0=0$. Hence, (3) implies (5). (c) Again assume $x \cdot C y=0$. By 1.1 (iii), $y \leqq C y$, so that $C x \cdot y \leqq C x \cdot C y$. But, by (5), $C x \cdot C y=0$. Hence, $C x \cdot y=0$. (d) Suppose $C x=x$, then $-x \cdot C x=0$. Using (6), it follows that $C-x \cdot x=0$, or $C-x \leqq-x$. But by 1.1 (iii), $-x \leqq C-x$. Therefore, $C-x=-x$.

(e) By 1.1 (iv), $C C x=C x$, so that by (4), $C-C x=-C x$; taking the complement of both sides of the preceding equation, we obtain (1). Q.E.D.

Our next theorem shows that the closure operator in a C-5 algebra is completely distributive over addition. (This, of course, is not necessarily true in a closure algebra.)

Theorem 1.3. If $A=\langle A, \cdot,-, C\rangle$ is a C-5 algebra, $B \subseteq A$, and $\sum_{x \in B} x$ is in $A$, then $C \sum_{x \in B} x=\sum_{x \in B} C x$. (Where, if $f$ is any function from $A$ to $A, \sum_{x \in B} f(x)=1$.u.b. $\{f(x): x \in B\}$. Similarly, $\prod_{x \in B} f(x)=$ g.l.b. $\{f(x): x \in B\}$.)

Proof. This theorem follows immediately from 1.2 (6) and Theorem 1.14 (ii) of Jónsson and Tarski [1]. If in Theorem 1.3 we replace $C$ by $I$ and $\sum$ by $\Pi$, we obtain the dual theorem and its proof is analogous.

2. MacNeille's extension. Let $A=\langle A, \cdot,-\rangle$ be a Boolean algebra. We can construct an extension $A_{e}=\left\langle A_{e}, \cdot_{e},-{ }_{e}\right\rangle$ of $A$ as follows: $x \in A_{e}$ if and only if $x \in A$ or $x$ is the least upper bound or greatest lower bound of a sub-set of $A$ (the method of Dedekind cuts). If $x$ and $y$ belong to $A_{e}$ then $x \cdot{ }_{e} y=\prod_{x \leqq x^{*} \in A, y \leqq y^{*} \in A} x^{*} \cdot y^{*}$ and $-{ }_{e} x$ $=\prod_{x \geqq x^{*} \in A}-x^{*}$. $\mathcal{A}_{\theta}$ is MacNeille's extension of $\mathcal{A}$ and it is known that $\mathcal{A}_{e}$ is a complete (i.e. all sums and products exist) Boolean algebra which preserves all sums and products of $\mathcal{A}^{6}{ }^{6}$ (Topologically, $\mathcal{A}_{\boldsymbol{\theta}}$ may be described as follows: because of Stone's Theorem, ${ }^{7}$ we may assume without loss of generality that the Boolean algebra $\mathcal{A}$ is a set-field consisting of all open and closed sets of a totally-discon-

- MacNeille [4, Theorems 11.9 and 11.13].

${ }^{7}$ Stone $[6$, Theorem 1$]$. 
nected compact space. Hence, $A$ is the set of all open and closed sets of the space, and - and - are the set theoretical operations of intersection and complementation. Then $A_{c}$ is the set of all regular open sets of the space. (I.e. $x$ is a regular open set if $x=$ interior of the closure of $x$.) If $x$ and $y$ belong to $A_{e}$, then $x \cdot e_{e} y=x \cdot y$ and $-{ }_{e} x=$ interior of $-x . c A_{e}=\left\langle A_{e}, \cdot_{e},-{ }_{e}\right\rangle$ is MacNeille's extension of

$$
A=\langle A, \cdot,-\rangle \text {.) }
$$

We may now define MacNeille's extension of a C-5 algebra as follows:

Definition 2.1. Let $A=\langle A, \cdot,-, C\rangle$ be a C-5 algebra. The algebra $A_{e}=\left\langle A_{e}, \cdot_{e},-_{e}, C_{e}\right\rangle$ is said to be MacNeille's extension of $\mathcal{A}$ if the following conditions are satisfied:

(i) $\left\langle A_{e}, \cdot_{e},-{ }_{e}\right\rangle$ is MacNeille's extension of the Boolean algebra $\langle A, \cdot,-\rangle$.

(ii) $C_{e} x=\prod_{x \leqq x^{*} \in A} C x^{*}$ for all $x$ in $A_{e}$.

It is a simple matter to verify that $A_{e}$ is a closure algebra. In the remaining part of this section we shall show that $A_{e}$ is a C-5 algebra.

Let $F$ be the set of all closed elements of $A$ (i.e. $x \in F$ if and only if $x \in A$ and $C x=x$ ). We assert:

$$
\prod_{x \leqq x^{*} \in A} C x^{*}=\prod_{x \leqq x^{*} \in F} x^{*} \text {, for all } x \text { in } A_{e} .
$$

For, suppose $y$ is a factor of $\prod_{x \leqq x^{*} \in F} x^{*}$, then $x \leqq y \in F \subseteq A$, so that $C y$ is a factor of $\prod_{x \leqq x^{*} \in A} C x^{*}$. But since $y \in F, C y=y$. Therefore, every factor of $\prod_{x \leqq x^{*} \in F} x^{*}$ is a factor of $\prod_{x \leqq x^{*} \in A} C x^{*}$. Now, suppose $y$ is a factor of $\prod_{x \leqq x^{*} \in A} C x^{*}$, then there exists an $x^{*}, x \leqq x^{*} \in A$, such that $y=C x^{*}$. Then, by 1.1 (iv), $C y=y$, so that $y \in F$, and by 1.1 (iii), $x^{*} \leqq C x^{*}$. Hence, $y$ is a factor of $\prod_{x \leqq x^{*} \in F} x^{*}$. Therefore, the factors of the left side and right side of equation (1) are identical.

Next, if $C_{\bullet} x=x$, we assert:

$$
\sum_{x \geqq x^{*} \in A} C x^{*}=\sum_{x \geqq x^{*} \in F} x^{*}
$$

because, suppose $y$ is a term of $\sum_{x \geqq x^{*} \in F} x^{*}$, then $x \geqq y \in F \subseteq A$, so that $C y=y$ is a term of $\sum_{x \geqq x^{*} \in A} C x^{*}$. If $y$ is a term of $\sum_{x \geqq x^{*} \in A} C x^{*}$, then there exists an $x^{*}, x \geqq x^{*} \in A$, such that $y=C x^{*}$. Then, by hypothesis and $1.1(\mathrm{v}), x=C_{e} x \geqq C x^{*}=y$. Moreover, by 1.1 (iv), $y \in F$. Hence, $y$ is a term of $\sum_{x \geqq x^{*} \in F} x^{*}$ and (2) holds.

Now, $x=\sum_{x \geqq x^{*} \in A} x^{*}$, so that by the definition of $\sum$,

$$
\sum_{x \geqq x^{*} \in F} x^{*} \leqq x
$$


and by 1.1 (iii), $x \leqq \sum_{x \geqq x^{*} \in A} C x^{*}$. Hence, it follows from (2) that if $C_{0} x=x$ then

$$
x=\sum_{x \geqq x^{*} \in F} x^{*} .
$$

Finally, if $C_{0} x=x$, we may calculate $C_{0}-{ }_{e} x$ as follows:

$$
\begin{aligned}
& C_{e}-{ }_{e} x=\prod_{-. x \leqq x^{*} \in A} C x^{*} \\
& =\prod_{-x \leqq x^{*} \in F} x^{*} \\
& \left.=\prod_{-{ }_{0} \leqq-x^{*} \in F}-x^{*} \quad \text { [substituting }-x^{*} \text { for } x^{*}\right] \\
& =\prod_{x \geqq x^{*} \in F}-x^{*} \quad \text { [using Boolean algebraic properties } \\
& \text { and } 1.2 \text { (4) for elements of } A \text { ] } \\
& =-。 \sum_{x \geqq x^{*} \in F} x^{*} \quad \text { [by Boolean algebraic properties] } \\
& =-e_{e} x
\end{aligned}
$$

Hence, it follows from 1.1 (vii) and 1.2 (4) that $A_{e}$ is a C-5 algebra, and we obtain the theorem:

TheOREM 2.2. If $A$ is a C-5 algebra and $A_{e}$ is MacNeille's extension of $\mathcal{A}$, then $\mathcal{A}_{0}$ is a complete C-5 algebra which preserves all sums and products of $A$.

REMARK. One of the consequences of Theorem 2.2 is that the results which Rasiowa [5] has obtained for Lewis' system S4 can immediately be extended to S5. ${ }^{8}$

\section{BIBLIOGRAPHY}

1. Bjarni Jónsson and Alfred Tarski, Boolean algebras with operators, Amer. J. Math. vol. 73 (1951) pp. 891-939.

2. C. I. Lewis and C. H. Langford, Symbolic logic, New York, Dover, 1932, 506 pp.

3. J. C. C. McKinsey and Alfred Tarski, The algebra of topology, Ann. Math. vol. 45 (1944) pp. 141-191.

4. H. M. MacNeille, Partially ordered sets, Trans. Amer. Math. Soc. vol. 42 (1937) pp. $416-460$.

5. H. Rasiowa, Algebraic treatment of the functional calculus of Heyting and Lewis, Fund. Math. vol. 38 (1951) pp. 99-126.

6. M. H. Stone, Applications of the theory of Boolean rings to general topology. Trans. Amer. Math. Soc. vol. 41 (1937) pp. 375-481.

\section{STANFORD UNIVERSITY}

${ }^{8}$ See footnote 2 for a reference for Lewis' systems S4 and S5. 